\title{
Efficacy and safety of topical herbal medicine treatment on recurrent aphthous stomatitis: a systemic review
}

This article was published in the following Dove Press journal:

Drug Design, Development and Therapy

31 December 2015

Number of times this article has been viewed

\author{
Chun-Lei Li ${ }^{1, *}$ \\ He-Long Huangl,* \\ Wan-Chun Wang ${ }^{2}$ \\ Hong Hua' \\ 'Department of Oral Medicine, Peking \\ University School and Hospital of \\ Stomatology, Beijing, ${ }^{2}$ Department \\ of Oral Medicine, Qingdao \\ Stomatological Hospital, Qingdao, \\ People's Republic of China \\ *These authors contributed equally \\ to this work
}

Correspondence: Hong Hua Department of Oral Medicine, Peking University School and Hospital of Stomatology, 22 Zhongguancun Avenue South, Haidian District, Beijing I0008I, People's Republic of China Tel +86 1082195218

Email honghua1968@aliyun.com

Wan-Chun Wang

Qingdao Stomatological Hospital, No 17 Dexian Road Shinan, Qingdao 26600I,

People's Republic of China

Tel +861082792425

Email kqwwch@I26.com

\begin{abstract}
This study aimed to evaluate the efficacy and safety of topical treatment with natural herbal medicines on recurrent aphthous stomatitis (RAS). Nine electronic databases were searched to identify the randomized controlled trials and clinical controlled trials that reported the potential effect of natural herbal medicines on RAS published in Chinese or English. Ulcer size and duration, and remission of pain were assessed as main outcome measures. The methodological quality of the studies was evaluated using the Cochrane Handbookfor Systemic Review of Interventions and Rev Man software. Thirteen trials with a total of 1,515 patients were included in the present analysis, which showed that topical treatment with natural herbal medicines seemed to benefit RAS patients by reducing ulcer size, shortening ulcer duration, and relieving pain without severe side effects. In conclusion, there is some evidence of the efficacy of topically applied natural herbal medicines with regards to improved RAS outcome measures and fewer side effects. However, given the limitations of this study, the evidence remains insufficient. Well-designed and high-quality randomized controlled trials are required for further exploration.
\end{abstract}

Keywords: recurrent aphthous stomatitis, herbal medicine, systemic review, randomized controlled trial, clinical controlled trials, oral disease

\section{Introduction}

Recurrent aphthous stomatitis (RAS), or recurrent aphthous ulcer (RAU), is one of the most common oral diseases, with an estimated prevalence of $25 \% .{ }^{1}$ Many factors may be involved in its progression, such as genetic predisposition, immunological abnormalities, microbial infection, psychological stress, and hormonal state. ${ }^{2}$ Since the etiology and pathogenesis of RAS remains unclear, there is currently no consensus regarding a definitive curative therapy. The commonly accepted treatment strategy is to lessen the pain and duration of lesions. ${ }^{3}$ Topical corticosteroids, antibiotics, and analgesics are highly recommended for patients with RAS. ${ }^{4}$ However, longer treatment and frequent exposure to these medications may cause fungal infection and drug resistance, which may further lead to more severe adverse effects or even life-threatening complications. ${ }^{5}$

Natural herbal medicines as an alternative therapy for RAS have been widely used in many countries for decades. ${ }^{6-8}$ Clinical studies on the use of such remedies have reported favorable benefits to patients by reducing the discomfort and duration of ulcers. ${ }^{8-11}$ However, no evidence-based reviews regarding the efficacy and safety of the topical application of these medicines on RAS have been available in the literature to date. Therefore, we conducted this analysis to evaluate the efficacy and safety of topical treatment with natural herbal medicines on RAS. 


\section{Materials and methods}

\section{Database and search strategy}

This study was performed in accordance with the Preferred Reporting Items for Systematic Reviews and Meta-analyses guidelines. ${ }^{12}$ All clinical trials that reported the effect of topical treatment with natural herbal medicines on RAS were selected in the Chinese National Knowledge Infrastructure, the Chinese Biomedical Literature Database, the Chinese Scientific Journal Database (VIP), WANFANG data (WANFANG), PubMed, Excerpta Medica dataBASE (EMBASE), Science Citation Index (SCI), Current Controlled Trials (CCT), and the Cochrane Central Register of Controlled Trials in the Cochrane Library (up to April 2015). Publication language was confined to Chinese and English. The following terms were searched individually or combined: "Chinese patent medicine", "Chinese patent drugs", "traditional Chinese medicine", "Chinese herbology", "Chinese medicine", "Chinese material medica", "Chinese herbs", "Chinese herbal medicine", "natural herbal medicine", "Chin Tradit Pat Med", "herbal medicine", "recurrent aphthous ulcer", "recurrent aphthous stomatitis", "recurrent oral ulcer", and "recurrent oral ulceration". Manual searching was used as a complementation.

\section{Inclusion and exclusion criteria}

\section{Inclusion criteria}

Randomized controlled trials (RCTs) and clinical controlled trials (CCTs) that evaluated the efficacy and safety of topical treatment with natural herbal medicines on RAS were collected. In accordance with "The diagnosis and management of recurrent aphthous stomatitis: a consensus approach", ${ }_{13}$ patients meeting the diagnostic criteria for RAS were included, with no restrictions on age, sex, or race. Patients in the experimental group received natural herbal medicines locally, without combined topical Western medications or systemic administration. Patients in the control group had to receive placebo treatment, chlorhexidine rinse. The outcome measures included the assessment of ulcer size, lesion duration, and remission of pain.

\section{Exclusion criteria}

Studies which there was duplication of study subjects were excluded, as were case reports or clinical observations without control groups, reviews, workshop summaries, translated papers or abstracts, animal studies, research reports without relevant or adequate information on participants, and interventions.

\section{Data extraction and quality assessment}

Two independent reviewers (CL Li and HL Huang) identified studies using the search strategy. If the eligibility of a study was not unanimous, a third reviewer party (H Hua and WC Wang) was consulted. Data were extracted from the included studies as follows: journal citation, author, year of publication, title, sample size, mediations, methodological details, therapeutic duration, and clinical outcomes.

The quality of the enrolled publications was assessed according to the Cochrane Handbook for Systemic Review of Interventions, Version 5.1.0, and Rev Man 5.3 software. ${ }^{14}$ The assessment criteria were random sequence generation (selection bias), allocation concealment (selection bias), blinding of participants and personnel (performance bias), blinding of outcome assessment (detection bias), incomplete outcome data (attrition bias), selective reporting (reporting bias), and other bias.

\section{Data analysis}

Due to the poor homogeneity of the included publications, only descriptive analysis was conducted in the present study.

\section{Results}

\section{Description of the assessed publications}

A total of 3,231 abstracts $(2,953$ in Chinese and 278 in English) were selected from nine databases; 1,154 studies were excluded for duplication. According to the exclusion criteria, screening of the titles and abstracts resulted in the removal of 1,948 articles. Full-text review of the remaining 129 publications indicated that 13 studies met the inclusion criteria and they were enrolled into the final analysis. ${ }^{8-11,15-23}$ The flowchart displayed in Figure 1 shows the detailed selection process.

To share more information with regard to this topic, part of excluded articles were provided as Supplementary material.

\section{Characteristics of the studies}

The characteristics of the 13 studies are summarized in Table 1. A total of 1,515 RAS patients were studied in this review. The sample size ranged from 15 to 150 patients in each study. Twelve different types of herbal medicine, in the form of gargles, membranes, powders, tablets, toothpastes, and gelatin preparations, were used, among which eight were traditional Chinese medicines and four were Iranian herbal medicines. The experimental period in these trials ranged from 3 to 10 days, with an average time of $5.92 \pm 2.21$ days.

\section{Risk of bias and quality assessment of the studies}

Among the included studies, 12 were RCTs and one was selfcontrolled. The quality of over $50 \%$ of enrolled studies was 


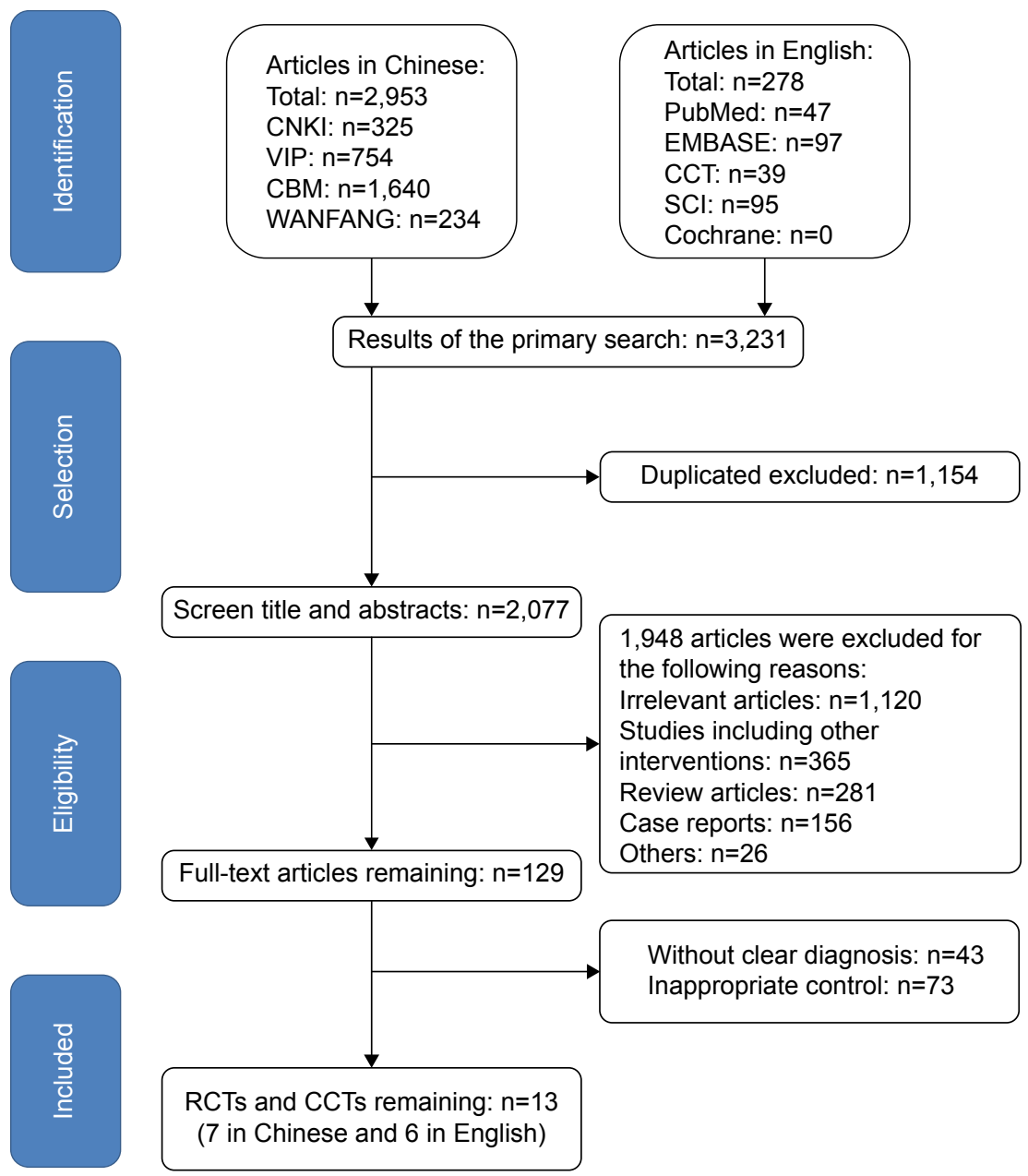

Figure I Study selection process.

Abbreviations: CBM, Chinese Biomedical Literature Database; CCTs, clinical controlled trials; CNKI, Chinese National Knowledge Infrastructure; EMBASE, Excerpta Medica database; RCTs, randomized controlled trials; VIP, the Chinese Scientific Journal Database; WANFANG, WANFANG data; SCI, Science Citation Index.

rated as poor, according to the Cochrane quality assessment criteria (Table 2 and Figure 2).

\section{Effects of the interventions}

Due to the heterogeneity of the enrolled trials, only descriptive analysis was conducted in this study.

\section{Main outcome measure}

The main outcome measures assessed in this study were ulcer size, lesion duration, and remission of pain.

\section{Size of ulcer}

Four out of 13 trials reported a change in ulcer size, which was measured by different methods. Haghpanah et a ${ }^{11}$ evaluated the average diameter of all ulcers; however, Jiang et $\mathrm{a}^{10}$ and $\mathrm{Liu}^{23}$ evaluated the maximum diameter of the ulcer, while Liu et $\mathrm{a}^{8}$ determined the size of the ulcer by the maximum diameter and its vertical diameter. Nevertheless, all the studies reported a statistically significant reduction of the ulcer size in patients receiving herbal medicine therapy compared with the controls, with the exception of the study by Haghpanah et al, ${ }^{11}$ who reported that a significant difference between the herbal medicine group and the control group was only observed in the first day of the intervention, with no statistically significant differences between the groups for the remaining time points.

\section{Duration of ulcer}

Four trials reported data on the duration of the ulcer. Ghalayani et $\mathrm{al}^{16}$ reported a significant difference in the mean healing time between $8.6 \pm 0.99$ days of placebo treatment and $5.3 \pm 0.81$ days of treatment with Punica granatum extract $(P<0.001)$. In the study by Liu et al, ${ }^{22}$ it was found that Tian-zhu aerosol oral rinse remarkably reduced the duration of the ulcer compared with chlorhexidine rinse $(P<0.05)$; however, no detailed information was described. In the study by Amanlou et al, ${ }^{15}$ the average time for complete healing of the lesions in patients receiving Satureja khuzestanica extract 


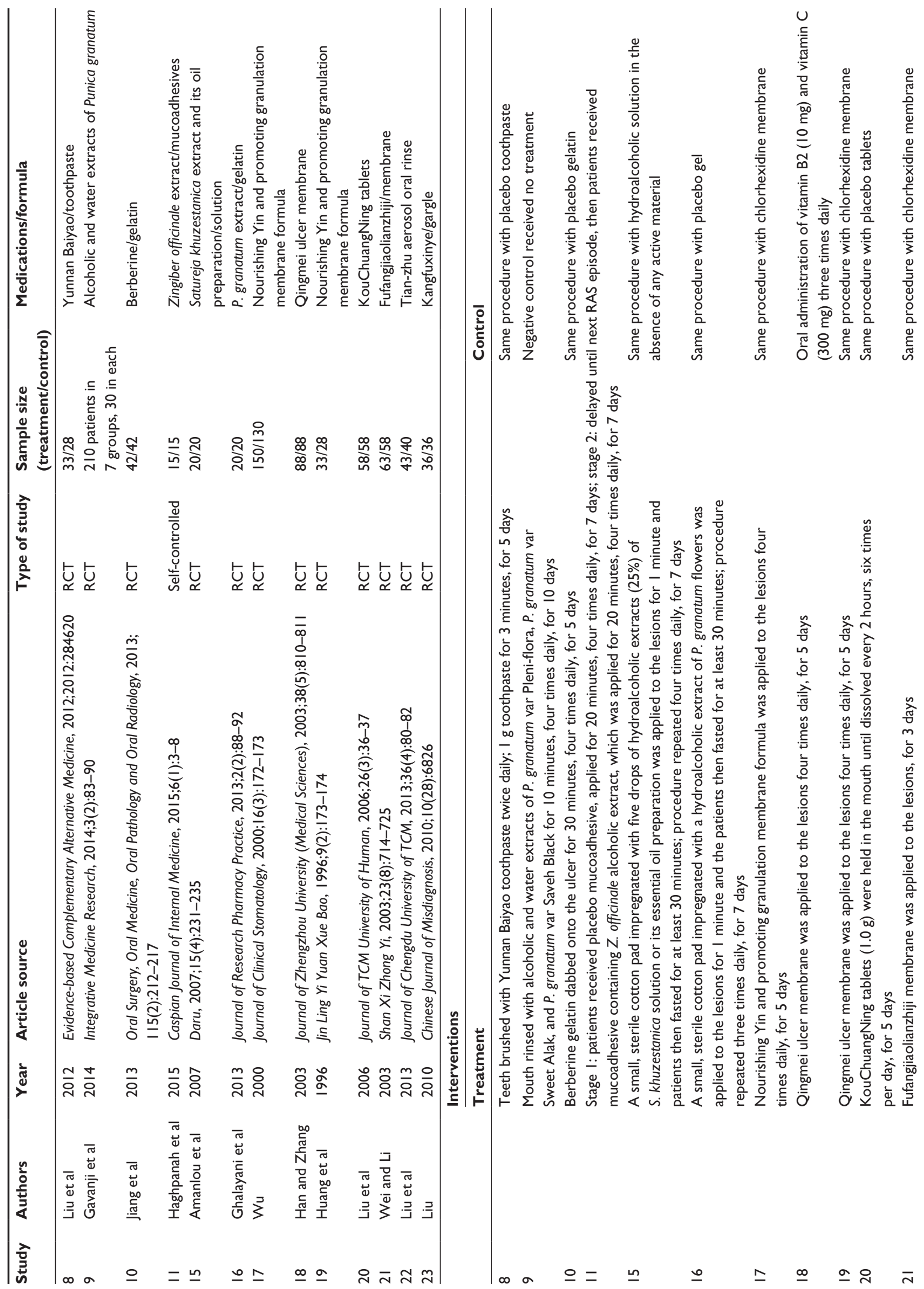




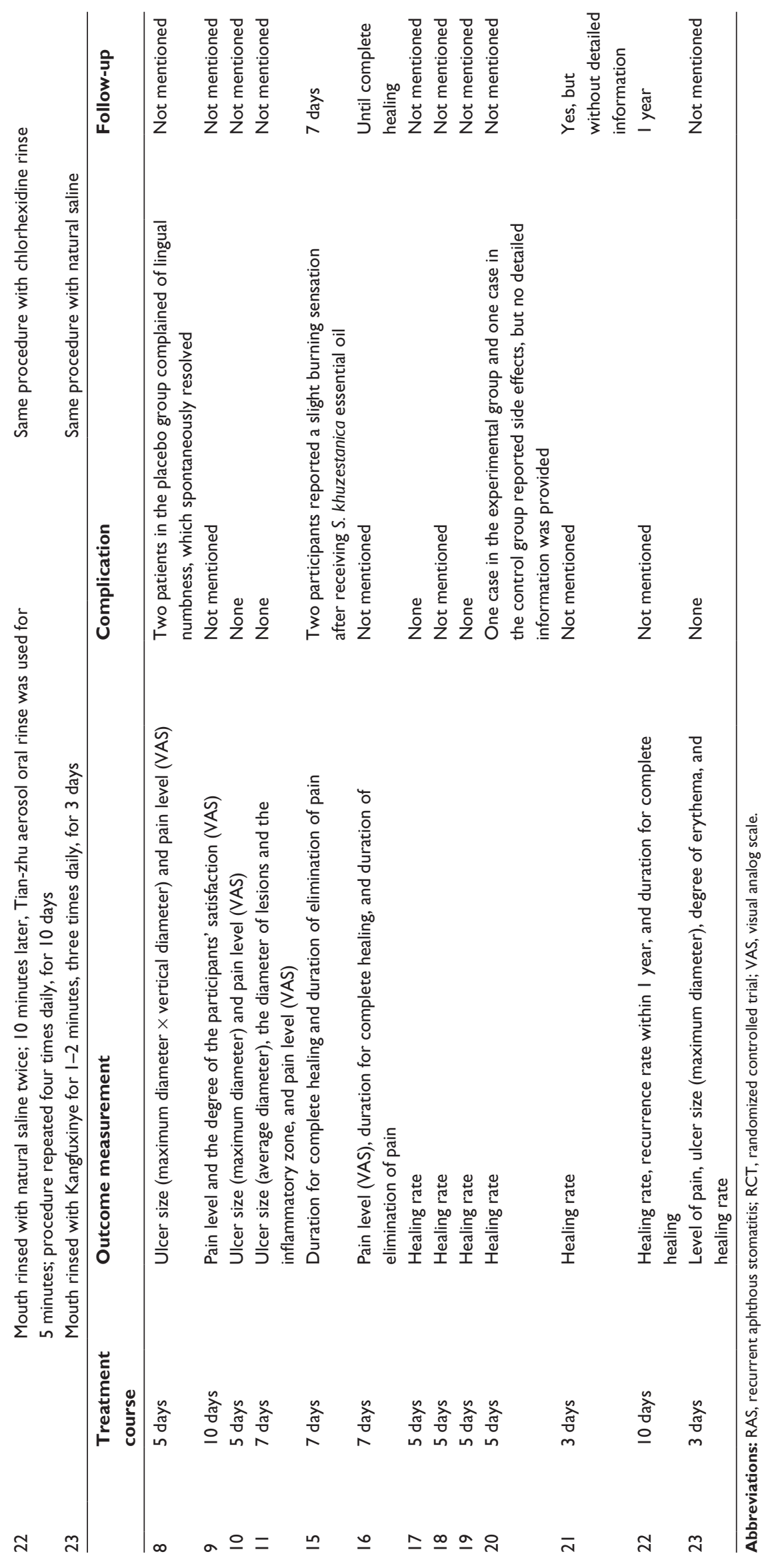




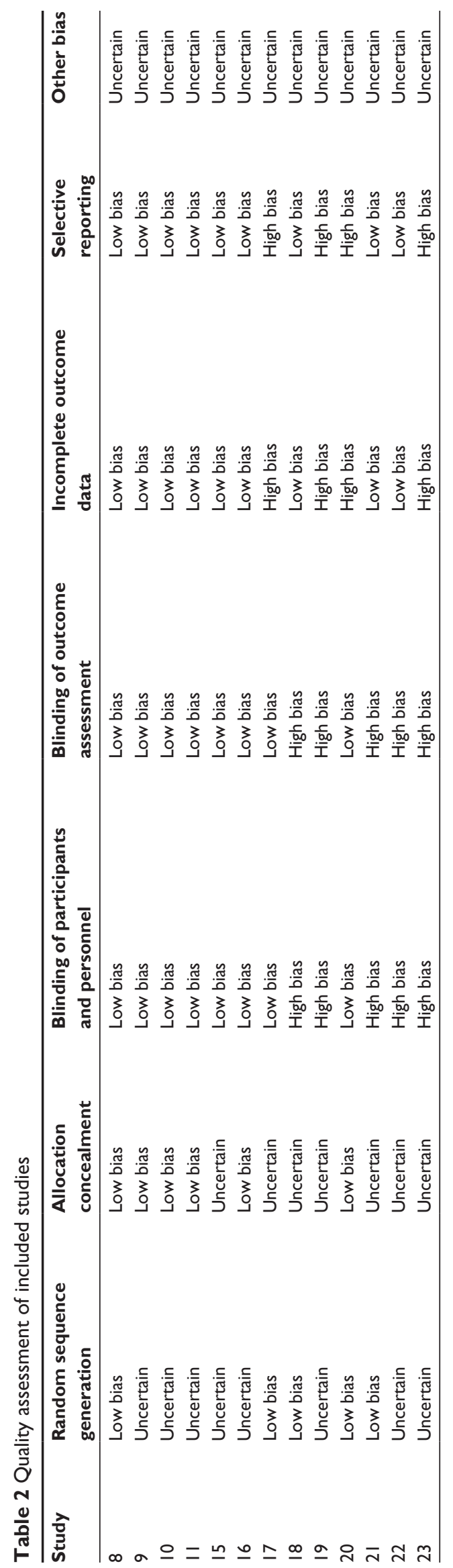

or its essential oil was $5.90 \pm 1.24$ days and $6.85 \pm 1.3$ days, respectively, which were both significantly less than those receiving placebo treatment ( $10.40 \pm 1.66$ days, $P=0.0001)$. Moreover, there was no significant difference between the experimental groups. In the study by Wei and $\mathrm{Li}^{21}$ the healing time of the lesions in patients receiving Fufangiiaolianzhiji ( $2.79 \pm 0.30$ days) was significantly shorter than when patients received a chlorhexidine rinse $(6.02 \pm 1.45$ days, $P<0.05)$.

\section{Remission of pain}

Remission of pain was described in all the trials included in this literature review and it was described as the main outcome index in six studies. In addition, a visual analog scale (VAS) was applied in five out of the six studies to record the level of pain. In these studies, the visual analog scale score was significantly decreased in the herbal medicine groups than in the control groups. ${ }^{8-11,16}$ In addition, two trials reported the average period of pain elimination, which was significantly shorter in patients receiving herbal medicine than placebo treatment. ${ }^{15,16}$

\section{Side effects}

Five trials did not report side effects, $, 16,18,21,22$ while five trials reported that there were no side effects ${ }^{10,11,17,19,23}$ and three trials reported slight side effects. ${ }^{8,15,20}$ In the study by Liu et al, ${ }^{20}$ one patient receiving herbal medicine and one patient in the control group reported side effects, but no detailed information was provided. In the study by Amanlou et al, ${ }^{15}$ two participants reported a slight burning sensation after receiving S. khuzestanica essential oil (prepared in an ethanol/water mixture: $50 \% \mathrm{v} / \mathrm{v}$ ) on the first application. Liu et al ${ }^{8}$ noted that two patients in the placebo group complained of slight lingual numbness, which spontaneously remedied.

\section{Discussion}

RAS is one of the most common oral disorders and its etiology is not well understood. Its management is mainly directed toward symptomatic, supportive treatment. ${ }^{3}$ Therefore, corticosteroids and analgesics serve as the first choice for RAS patients. ${ }^{4}$ However, longer treatment times and frequent exposure to such drugs may induce severe complications, such as secondary fungal infections and drug resistance. ${ }^{5}$ There has been a long history of the use of natural herbal medicines for various disorders, including RAS, worldwide and such remedies have been studied both in clinic trials and experimental studies. ${ }^{8,9,24,25}$ In this study, we focused on the efficacy and safety of the topical application of natural herbal medicines for the treatment of RAS. 
Random sequence generation (selection bias)

Allocation concealment (selection bias)

Blinding of participants and personnel (performance bias)

Blinding of outcome assessment (detection bias) Incomplete outcome data (attrition bias)

Selective reporting (reporting bias)

Other bias
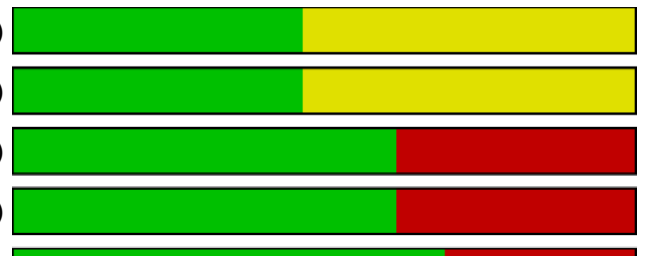

$$
\square
$$

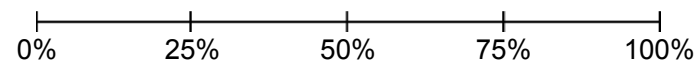

$\square$ Low risk of bias $\square$ Unclear risk of bias $\square$ High risk of bias

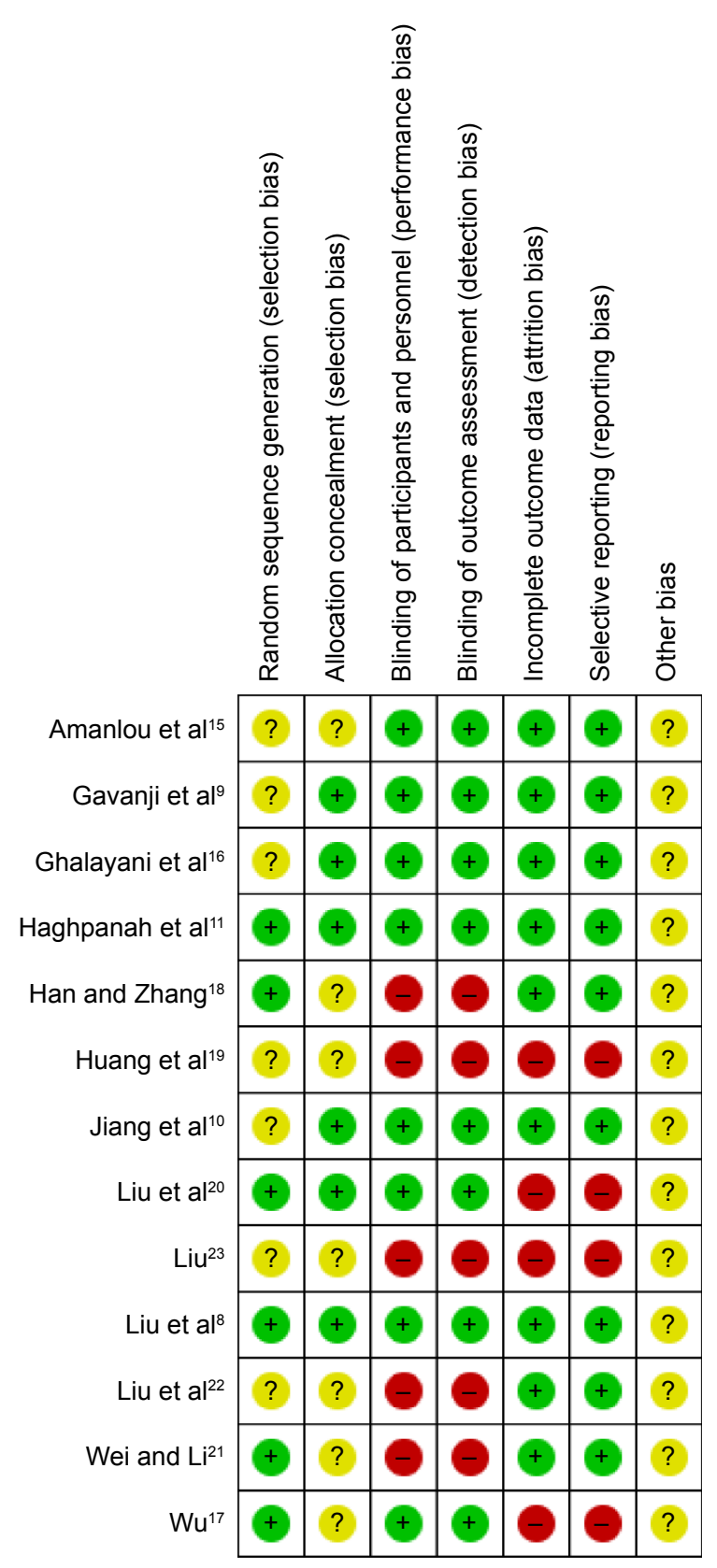

Figure 2 The Cochrane Collaboration's tool for assessing risk of bias. Note: +, low bias; ?, uncertain; -, high bias. 
A total of 1,515 subjects in 13 clinical trials were analyzed in the present analysis. Compared with controls, topical natural herbal medicines greatly improved the patients' symptoms by reducing ulcer size, shortening ulcer duration, and relieving pain without severe complications. However, only a weak conclusion can be drawn due to several limitations. First, the homogeneity of the studies was quite poor, with variables such as the various types of treatment, dosage, formula, application method, sample size, and experiment duration. Second, the quality of the studies was not sufficient because of poor study design and high risks in the performance, detection, attrition, and reporting bias. Therefore, a meta-analysis could not be conducted based on the current data. Consequently, this analysis unveiled the need for well-designed multicenter RCTs, which are of paramount importance for further exploration. Furthermore, precise criteria and standard methodologies should be established to ensure high-quality data.

The rationale of study designs can be a guarantee of strong clinical trials. The herbal medicine-oriental model or disease-oriental model may greatly affect the inclusion and exclusion criteria of future meta-analyses.

Detailed information of the herbal medicine used in future studies, including the formula, dosage, therapy duration, application protocols, and control intervention, should be provided. If possible, the dosage and application protocol should be homogenized for different forms of the same medicine.

In the studies included in this review, the therapy duration ranged from 3 to 10 days. However, we found that the natural course of an untreated ulcer was $9.5 \pm 1.3$ days ${ }^{15,16}$ and the duration of herbal medicine treatment was $5.21 \pm 1.73$ days. ${ }^{15,16,21,22}$ It is therefore suggested that the experimental period should exceed 7 days to avoid missing valuable data. Moreover, follow-up is highly recommended.

In addition to the above, the index and measurement of outcomes should be described clearly and consistently in future studies. According to the current data, ulcer size, lesion duration, and level of pain are commonly considered as the main outcome indicators. However, researchers often failed to assess them in a standard way, especially with regards to the ulcer size. Actually, manifestation of RAS may be a single ulcer or several round or elliptic recurrent ulcers in the oral mucosa. ${ }^{4}$ Therefore, measurement of the maximum diameter may not be suitable to determine the ulcer size accurately. Theoretically, evaluation of the ulcer area may be better in describing the lesion size; however, the precise calculation may restrict its application in clinic. We suggest that measurement of the maximum diameter and its vertical diameter should be made, as described in the study by Liu et $\mathrm{al},{ }^{8}$ as it is a precise and convenient way to determine the size of lesions. Finally, appropriate statistical methods also represent an important part of study protocols and can allow sample size calculation and data analysis.

In summary, the current data show favorable benefits of the topical treatment of RAS with natural herbal medicines and only three of the included studies reported slight and/or transient side effects during the clinical trial period. Thus, there is some evidence to suggest that topical herbal medicine therapy is an effective and safe alternative option to current Western medicine-based treatments for RAS.

\section{Conclusion}

There is weak evidence with regards to the efficacy of the topical application of natural herbal medicines for the treatment of RAS patients' conditions, with few side effects reported. However, given the limitations of the trials included in this assessment and the methodologies employed in the current data, a definitive conclusion cannot be drawn. Welldesigned and high-quality RCTs are required for further exploration.

\section{Acknowledgment}

This work was supported by the National Key Clinic Program (2013).

\section{Disclosure}

The authors report no conflicts of interest in this work.

\section{References}

1. Scully C, Porter S. Oral mucosal disease: recurrent aphthous stomatitis. Br J Oral Maxillofac Surg. 2008;46(3):198-206.

2. Greenbery M, Glick M. Burket's Oral Medicine Diagnosis and Treatment. 11th ed. Hamilton: BC Decker; 2008:63-65.

3. Porter SR, Hegarty A, Kaliakatsou F, Hodgson TA, Scully C. Recurrent aphthous stomatitis. Clin Dermatol. 2000;18(5):569-578.

4. Femiano F, Lanza A, Buonaiuto C, et al. Guidelines for diagnosis and management of aphthous stomatitis. Pediatr Infect Dis J. 2007;26(8): 728-732.

5. Eisen D, Carrozzo M, Bagan Sebastian JV, Thongprasom K, Number V. Oral lichen planus: clinical features and management. Oral Dis. 2005; 11(6):338-349.

6. McBride DR. Management of aphthous ulcers. Am Fam Physician. 2000; 62(1):149-154, 160

7. Rattan J, Schneider M, Arber N, Gorsky M, Dayan D. Sucralfate suspension as a treatment of recurrent aphthous stomatitis. J Intern Med. 1994;236(3):341-343.

8. Liu X, Guan X, Chen R, Hua H, Liu Y, Yan Z. Repurposing of yunnan baiyao as an alternative therapy for minor recurrent aphthous stomatitis. Evid Based Complement Alternat Med. 2012;2012:284620.

9. Gavanji S, Larki B, Bakhtari A. The effect of extract of Punica granatum var. pleniflora for treatment of minor recurrent aphthous stomatitis. Integr Med Res. 2014;3(2):83-90. 
10. Jiang XW, Zhang Y, Zhu YL, et al. Effects of berberine gelatin on recurrent aphthous stomatitis: a randomized, placebo-controlled, double-blind trial in a Chinese cohort. Oral Surg Oral Med Oral Pathol Oral Radiol. 2013;115(2):212-217.

11. Haghpanah P, Moghadamnia AA, Zarghami A, Motallebnejad M. Muco-bioadhesive containing ginger officinale extract in the management of recurrent aphthous stomatitis: a randomized clinical study. Caspian J Intern Med. 2015;6(1):3-8.

12. Moher D, Liberati A, Tetzlaff J, Altman DG, Group P. Preferred reporting items for systematic reviews and meta-analyses: the PRISMA statement. Int J Surg. 2010;8(5):336-341.

13. Scully C, Gorsky M, Lozada-Nur F. The diagnosis and management of recurrent aphthous stomatitis: a consensus approach. J Am Dent Assoc. 2003;134(2):200-207.

14. Higgins JPT, Green S. Cochrane Handbook for Systemic Reviews of Interventions, Version 5.1.0. 2011. The Cochrane Collaboration. Available from: http://handbook.cochrane.org/. Accessed January 5, 2015.

15. Amanlou M, Babaee N, Saheb-Jamee M, Salehnia A, Farsam H, Tohidast Akrad Z. Efficacy of Satureja khuzistanica extract and its essential oil preparations in the management of recurrent aphthous stomatitis. Daru. 2007;15(4):231-235.

16. Ghalayani P, Zolfaghary B, Farhad AR, Tavangar A, Soleymani B. The efficacy of Punica granatum extract in the management of recurrent aphthous stomatitis. J Res Pharm Pract. 2013;2(2):88-92.
17. Wu GY. The effect of treating ROU with the membrane of nourishing yin and promoting granulation. J Clin Stomatol. 2000;16(3):172-173.

18. Han F, Zhang ZJ. Effect of qingmei ulcer membrance on recurrent oral ulcer in 88 cases. J Zhengzhou University: Med Sci. 2003;38(5): 810-811.

19. Huang HF, Xu X, Meng ZY, Chen L. Effect of the membrance of nourishing yin and promoting granulation on recurrent aphthous stomatitis. Jin Ling Yi Yuan Xue Bao. 1996;9(2):173-174.

20. Liu YP, Zhang L, Chen SJ. Clinical studies of the effect of KouChuangNing tablets on recurrent aphthous stomatitis with syndrome of accumulated heat in heart and spleen. J TCM Univ Human. 2006;26(3):36-37.

21. Wei QW, Li WD. Effect of Fufangjiaolianzhiji on recurrent oral ulcer in 63 cases. Shan Xi Zhong Yi. 2003;23(8):714-725.

22. Liu YX, Liu X, Li H, Yu F, Zheng XY, Huang ZY. Observation on clinical therapeutic efficacy of tian-zhu aerosol oral rinse treatment of 83 cases with oral ulcen. J Chengdu Univ TCM. 2013;36(4):80-82.

23. Liu GL. Effect of kangfuxinye on recurrent oral ulcer: a clinic observation. Chin J Mis Diagn. 2010;10(28):6826.

24. Zheng LW, Hua H, Cheung LK. Traditional Chinese medicine and oral diseases: today and tomorrow. Oral Dis. 2011;17(1):7-12.

25. Li CL, He J, Li ZG, Zheng LW, Hua H. Effects of total glucosides of paeony for delaying onset of Sjogren's syndrome: an animal study. J Craniomaxillofac Surg. 2013;41(7):610-615.
Drug Design, Development and Therapy

\section{Publish your work in this journal}

Drug Design, Development and Therapy is an international, peerreviewed open-access journal that spans the spectrum of drug design and development through to clinical applications. Clinical outcomes, patient safety, and programs for the development and effective, safe, and sustained use of medicines are a feature of the journal, which

\section{Dovepress}

has also been accepted for indexing on PubMed Central. The manuscript management system is completely online and includes a very quick and fair peer-review system, which is all easy to use. Visit http://www.dovepress.com/testimonials.php to read real quotes from published authors.

Submit your manuscript here: http://www.dovepress.com/drug-design-development-and-therapy-journal 